\title{
A Meta-Analysis of Lymphatic Vessel Invasion Correlated with Pathologic Factors in Invasive Breast Cancer
}

\author{
Sandi Shen ${ }^{1,2}$, Shizhen Zhong1, Hai Lu², Wenhua Huang1* ${ }^{*}$, Gaofang Xiao ${ }^{3}$ \\ ${ }^{1}$ Department of Anatomy, Academic College, Southern Medical University, Guangzhou, China \\ ${ }^{2}$ Headneck and Breast Surgery, Yuebei People Hospital, Shantou University, Shaoguan, China \\ ${ }^{3}$ Department of Pathology, Yuebei People Hospital, Shantou University, Shaoguan, China \\ Email: huangwenhua2009@139.com
}

Received 13 March 2015; accepted 6 April 2015; published 9 April 2015

Copyright (C) 2015 by authors and Scientific Research Publishing Inc.

This work is licensed under the Creative Commons Attribution International License (CC BY).

http://creativecommons.org/licenses/by/4.0/

(c) (i) Open Access

\section{Abstract}

Objectives: The invasive breast cancer is divided into four clinical subtypes: Luminal A-like, Luminal B-like, HER-2 positive, and triple-negative according to the expression status of estrogen receptor (ER), progesterone receptor(PR), human epidermal growth factor receptor-2 (HER-2) and Ki-67. The prognosis and treatment strategy vary with subtypes. The current studies have reported the relation between lymphatic vessel invasion (LVI) and the expression status of ER, PR, HER-2, Ki-67 in invasive breast cancer, but the results were debatable. So the meta-analysis was conducted to confirm the relation between LVI and the four factors. Methods: Literature was searched by entering the terms: breast AND (neoplasm OR cancer OR carcinoma) AND (lymphovascular OR "lymph vessel" OR "lymphatic vessel" invasion OR carcinoma embolus) AND (ER OR estrogen receptor OR PR OR progesterone receptor OR HER-2 OR human epidermal growth factor receptor-2 OR Ki-67 OR clinicopathological) in Pubmed. The merged odds ratio (OR) and 95\% confidence interval (CI) were estimated using fixed-effect model. Review Manager 5.2 was used to analysis the relation between LVI and the expression status of ER, PR, HER-2, Ki-67 in invasive breast cancer respectively. The fail-safe number was used to estimate publication bias. Results: The analysis included 5 studies, LVI positive rate was significant lower in ER positive, PR positive, HER-2 negative, low Ki-67 expression group statistically. The OR and 95\% CI were $0.6(0.44-0.81)$, $0.64(0.43$ - 0.95), 1.52(1.03 - 2.24), 5.29(1.53 - 18.35) respectively. Conclusions: LVI was significantly correlated with the expression status of ER, PR, HER-2 and Ki-67 in invasive breast cancer. Furthermore, LVI was consistent with poor prognostic expression status of the four factors.

\footnotetext{
${ }^{*}$ Corresponding author.
} 
Keywords

\section{Lymphatic Vessel Invasion, Estrogen Receptor, Progesterone Receptor, Human Epidermal Growth Factor Receptor-2, Ki-67, Breast Cancer}

\section{Introduction}

Breast cancer is a common cancer in female and one of the leading causes of cancer death in women. It accounts for $29 \%$ of all female new cancers and $15 \%$ of all female deaths due to cancers [1].

LVI is a key step of tumor cells reaching lymph node, therefore LVI is known as an independent predictor of lymph node metastases. Lymph node-positive breast cancer has a poor prognosis. Most studies have found that LVI is a promising predictor, but the results are often conflicting. In breast cancer, LVI wis presented as an independent predictor of disease-free survival (DFS) as well as overall survival (OS) [2] [3]. The 2005 St. Gallen consensus guidelines suggested LVI was recognized as one of the factors upon which to base treatment plan decisions [4].

The diagnosis of LVI was made based on the presence of tumor emboli within vessel lined by a single layer of endothelial cells without red blood cells. The monoclonal antibody D2-40 has often been used as a marker of lymphatic endothelium to identify tumor emboli in lymph vessels [5]. D2-40 stained the endothelium of lymphatic vessels and was useful and reliable in detecting LVI in invasive breast cancer. D2-40 staining showed that very few lymph vessels were present within the tumor mass and most of the lymphatic vessels were peritumoral.

Breast cancer was a heterogeneous disease, encompassing a number of distinct biological characters. The invasive breast cancer were divided into four clinic subtypes: Luminal A-like (ER and PR positive and HER-2 negative and low Ki-67), Luminal B-like (ER positive and HER-2 negative, and at least one of: high Ki-67; PR negative or low) or (ER or PR and HER-2 positive), HER-2 positive (HER-2 positive, ER and PR negative), and triple- negative (ER and PR and HER-2 negative) according to the expression status of ER, PR, HER-2, Ki-67. The prognosis and treatment strategy varied with subtypes. The current studies reported the relation between LVI and the four factors in invasive breast cancer, but the results were debatable [6]-[10]. So this meta-analysis was conducted to confirm the correlation between LVI and the expression status of ER, PR, HER-2, Ki-67.

\section{Materials and Methods}

\subsection{Literature Search Strategy}

Literature was searched by entering the terms: breast AND (neoplasms OR cancer OR carcinoma) AND (lymphovascular OR “lymph vessel” OR "lymphatic vessel” invasion OR carcinoma embolus) AND (ER OR PR OR estrogen receptor OR progesterone receptor OR HER-2 OR human epidermal growth factor receptor-2 OR Ki-67 OR clinicopathological) in Pubmed. The publish time of literature was unlimited. Only literature written in English language was included.

\subsection{Inclusion Criteria}

All of the following criteria had to be included in literature for this analysis: 1) Patients with breast cancer were not undergone radiotherapy, hormone therapy and chemotherapy before the pathological specimen were extracted; 2) The stages of the disease were $\mathrm{T}_{1-4} \mathrm{~N}_{0-3} \mathrm{M}_{0-1}$ or I-IV stages; 3 ) Hormone receptor status and Ki-67 were determined by hematoxylin and eosin (H \& E) staining and immunohistochemical (IHC) analysis. HER-2 status was determined by an additional in situ hybridization (ISH) only when the result was equivocal. LVI was identified by IHC analysis with monoclonal antibody D2-40; 4) All literature in English language.

\subsection{Exclusion Criteria}

The literature in which the detection method of the LVI was undefined or HE or from which the interested numbers cannot be extracted was excluded. 


\subsection{Data Extraction}

The following information was extracted from each eligible literature: authors' names, year of publication, expression status of ER, PR, HER-2, Ki-67 and LVI positive rate in each group.

\subsection{Statistical Analysis}

Rev Man 5.2 software was used to perform the meta-analysis. The odds ratio (OR) and 95\% confidence interval (CI) were used to estimate the correlation of LVI and pathological factors in the invasive breast cancer. The Mantel-Haenszel method was used to combine the ORs for the outcomes. The fixed-effect model was used to calculate the pooled outcomes if the heterogeneity was not present. Each study was weighted according to the sample size. The heterogeneity among studies was defined significant when $P<0.1$ for $\chi^{2}$ test or $\mathrm{I}^{2}>50 \%$. Fail-safe number was used for detecting publication bias according to the formula $N_{f 50.05}=(\Sigma Z / 1.64)^{2}-K$.

\section{Results}

\subsection{Eligible Literatures}

The searching deadline was Dec $26^{\text {th }}$, 2014. A total of 425 articles were identified from Pubmed, 17 articles were remained after exclusion based on the titles and abstracts. 1 duplication, 2 original articles in Chinese language, 1 original article in Portuguese language, 2 articles that could not provided interested data, 3 articles in which LVI was detected by HE and 3 original full texts that could not be obtained were removed. A total of 5 studies met the inclusion criteria for meta-analysis finally (Figure 1).

\subsection{Characteristics of Included Studies}

The final 5 studies were appraised critically. The 5 studies were published from 2007 to 2014 (Table 1). The judgement criterion of the ER, PR, HER-2 positive were not the same among the 5 studies. In the study of [Gujam FJ 2014], ER and PR status were assessed according to College of American Pathologists (CAP) guideline with cut-off value of $1 \%$ positive tumor nuclei. HER-2 status was assessed using microarrays. i.e. score 0 and $1+$ were regarded as negative; 2+ was regarded as equivocal, leading to referral for HER-2 In Situ Hybridization (ISH); 3+ was regarded as positive. LVI was identified by tumor cells within D2-40 positively stained vessels. In the study of [Braun M 2008], invasive tumors were judged to be receptor positive if either ER or PR was at least 1/12 (Remmele Score), the method and scoring system of the DAKO Hercep was used to test HER-2 status,

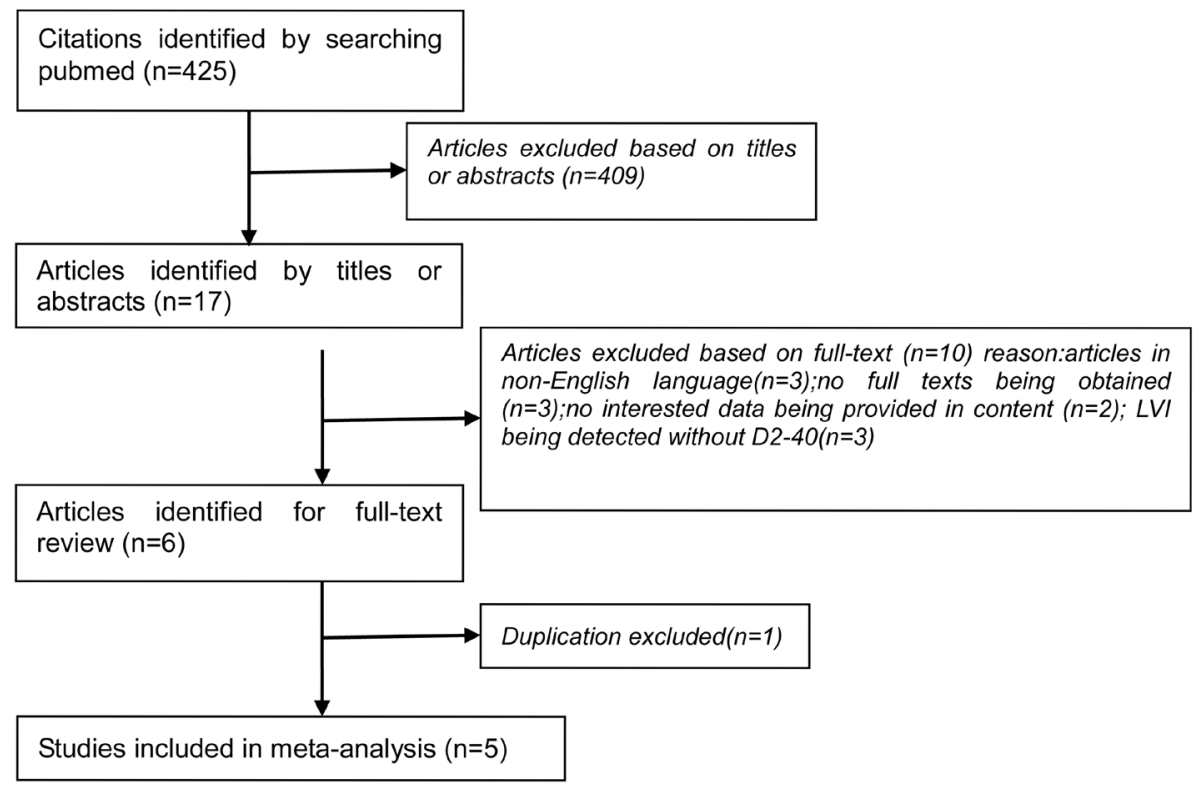

Figure 1. The flowchart of literature search. 
Table 1. LVI positive rates in different expression status of ER, PR, HER-2 and Ki-67 in invasive breast cancer in included studies.

\begin{tabular}{ccccccccc}
\hline \multirow{2}{*}{ Citations } & \multicolumn{7}{c}{ LVI Positive Rates } \\
\cline { 2 - 8 } & ER (+) & ER (-) & PR (+) & PR (-) & HER-2 (+) & HER-2 (-) & High Ki-67 & Low Ki-67 \\
\hline Braun M (2008) & $52 / 212$ & $18 / 42$ & & & $16 / 40$ & $54 / 214$ & & \\
Gujam FJ (2014) & $58 / 189$ & $69 / 171$ & $51 / 166$ & $76 / 194$ & $31 / 71$ & $96 / 289$ & & \\
Lee JA (2011) & $2 / 51$ & $6 / 29$ & $3 / 45$ & $5 / 35$ & $2 / 24$ & $6 / 56$ & $6 / 26$ & $1 / 17$ \\
Tezuka K (2007) & $27 / 68$ & $28 / 64$ & & & & & & $4 / 14$ \\
Widodo I (2013) & $16 / 24$ & $19 / 24$ & $18 / 34$ & $10 / 14$ & $21 / 34$ & $9 / 14$ & $27 / 34$ & 4 \\
\hline
\end{tabular}

(+): positive; (-): negative. ER: estrogen receptor; PR: progesterone receptor; HER-2: human epidermal growth factor receptor-2; LVI: lymphatic vessel invasion.

HER-2 was classified as negative if DAKO Score was 0 or $1+$ and as positive if Dako Score was $3+$. In cases of DAKO Score 2+, a ISH test was performed. In the paper of [Lee JA 2011], ER and PR status were assessed by standard IHC methods and were considered positive if the nuclear staining value was $\geq 10 \%$. The cut-off value of high Ki-67 expression was $\geq 20 \%$. The judgement standards were not mentioned in [Tezuka K 2007]. The judgement criterion of the ER, PR, HER-2 positive were the same as that in [Widodo I 2013], while the cut-off value of high Ki-67 expression was $\geq 10 \%$.

\subsection{Correlation of LVI with ER, PR, HER-2, Ki-67 in Invasive Breast Cancer}

LVI positive rates between ER positive and negative groups in invasive breast cancer were compared in 5 studies. There was no significant heterogeneity $\left(\mathrm{I}^{2}=11 \%, P=0.35\right)$. In the fixed-effect model there was statistically significant difference between ER positive and negative group (OR $=0.6,95 \%$ CI: $0.44-0.81, P=0.001)$ (Figure 2), which suggested LVI positive rate was low in ER positive group.

Due to significant heterogeneity $\left(\mathrm{I}^{2}=90 \%, P<0.01\right)$, the study of [Braun M 2008] was excluded. LVI positive rate between PR positive and negative groups in invasive breast cancer were compared in left 3 studies. There was no significant heterogeneity $\left(\mathrm{I}^{2}=0 \%, P=0.72\right)$. In the fixed-effect model there was statistically difference between PR positive and negative groups (OR $=0.64,95 \%$ CI: $0.43-0.95, P=0.03$ ) (Figure 3), which suggested LVI positive rate was low in PR positive group.

LVI positive rates between HER-2 positive and negative groups in invasive breast cancer were compared in 4 studies. There was no significant heterogeneity $\left(\mathrm{I}^{2}=0, P=0.61\right)$. In the fixed-effect model there was statistically difference between positive and negative HER-2 groups (OR = 1.52, 95\% CI: $1.03-2.24, P=0.04$ ) (Figure 4), which suggested LVI positive rate was low in HER-2 negative group.

LVI positive rates between high and low Ki-67 expression groups in invasive breast cancer were compared in 2 studies. There was no statistically heterogeneity $\left(\mathrm{I}^{2}=41 \%, P=0.19\right)$. In the fixed-effect model there was statistically difference between high and low Ki-67 expression groups (OR $=5.29,95 \%$ CI: $1.53-18.35, P=0.009$ ) (Figure 5), which suggested LVI positive rate was low in low Ki-67 expression group.

\subsection{Evaluation of Publication Bias}

As reports on LVI detected by D2-40 are rare in breast cancer, the publication bias can't be totally excluded. The publication bias were not visualized by funnel plot due to fewer articles, the fail-safe number could demonstrate that the publication bias existed probably in PR, Her-2 and Ki-67 sub-analysis in this meta-analysis. Adding articles maybe enhance the accuracy and reduce the publication bias in the future.

\section{Discussion}

The combined results indicated that LVI was correlated with the expression status of ER, PR, HER-2 and Ki-67 respectively in invasive breast cancer. And the LVI could act as a predictor of poor prognosis for invasive breast cancer.

Breast cancer with ER or PR positive is an indication for endocrine therapy, its prognosis is better than breast 


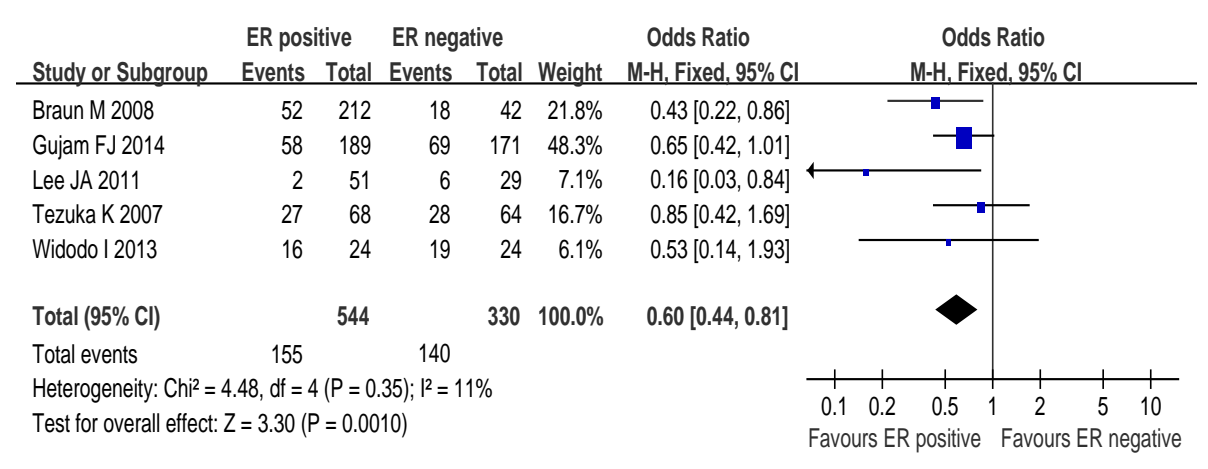

Figure 2. LVI positive rates between ER positive and negative groups in invasive breast cancer.

\begin{tabular}{|c|c|c|c|c|c|c|c|}
\hline Study or Subgroup & $\begin{array}{l}\text { PR posi } \\
\text { Events }\end{array}$ & $\begin{array}{l}\text { ive } \\
\text { Total }\end{array}$ & $\begin{array}{l}\text { PR nega } \\
\text { Events }\end{array}$ & $\begin{array}{l}\text { ative } \\
\text { Total }\end{array}$ & Weight & $\begin{array}{l}\text { Odds Ratio } \\
\text { M-H, Fixed, } 95 \% \mathrm{Cl}\end{array}$ & $\begin{array}{c}\text { Odds Ratio } \\
\text { M-H, Fixed, } 95 \% \mathrm{Cl}\end{array}$ \\
\hline Gujam FJ 2014 & 51 & 166 & 76 & 194 & $80.3 \%$ & $0.69[0.44,1.07]$ & \\
\hline Lee JA 2011 & 3 & 45 & 5 & 35 & $8.7 \%$ & $0.43[0.10,1.93]$ & \\
\hline Widodo I 2013 & 18 & 34 & 10 & 14 & $11.0 \%$ & $0.45[0.12,1.72]$ & \\
\hline Total $(95 \% \mathrm{Cl})$ & & 245 & & 243 & $100.0 \%$ & $0.64[0.43,0.95]$ & \\
\hline Total events & 72 & & 91 & & & & \\
\hline \multicolumn{7}{|c|}{$\begin{array}{l}\text { Heterogeneity: } \text { Chi }^{2}=0.64, d f=2(P=0.72) ; 1^{2}=0 \% \\
\text { Test for overall effect: } Z=2.19(P=0.03)\end{array}$} & $\begin{array}{ccccccc}0.1 & 0.2 & 0.5 & 1 & 2 & 5 & 10 \\
\text { Favours } & \text { PR positive } & \text { Favours PR negative }\end{array}$ \\
\hline
\end{tabular}

Figure 3. LVI positive rates between PR positive and negative groups in invasive breast cancer.

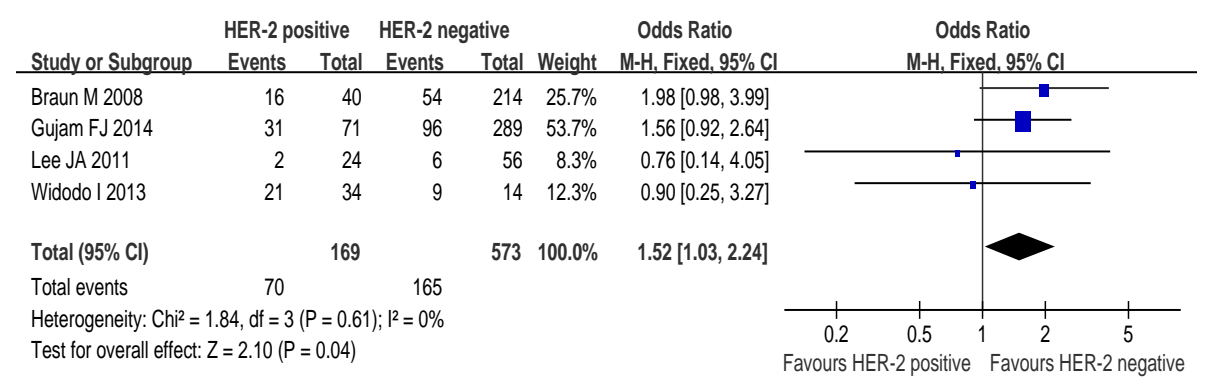

Figure 4. LVI positive rates between HER-2 positive and negative groups in invasive breast cancer.

\begin{tabular}{|c|c|c|c|c|c|c|c|}
\hline Study or Subqroup & $\begin{array}{l}\text { high Ki } \\
\text { Events }\end{array}$ & & $\begin{array}{l}\text { low Ki- } \\
\text { Events }\end{array}$ & & & $\begin{array}{l}\text { Odds Ratio } \\
\text { M-H. Fixed, } 95 \% \mathrm{Cl}\end{array}$ & $\begin{array}{c}\text { Odds Ratio } \\
\text { M-H. Fixed, } 95 \% \mathrm{Cl}\end{array}$ \\
\hline Lee JA 2011 & 6 & 62 & 1 & 17 & $54.9 \%$ & $1.71[0.19,15.30]$ & \\
\hline Widodo I 2013 & 27 & 34 & 4 & 14 & $45.1 \%$ & $9.64[2.32,40.16]$ & \\
\hline Total (95\% Cl) & & 96 & & 31 & $100.0 \%$ & $5.29[1.53,18.35]$ & \\
\hline Total events & 33 & & 5 & & & & \\
\hline $\begin{array}{l}\text { Heterogeneity: } \mathrm{Chi}^{2}= \\
\text { Test for overall effect: }\end{array}$ & $\begin{array}{l}70, \mathrm{df}=1 \\
=2.63(\mathrm{P}\end{array}$ & $\begin{array}{l}(P=0 \\
=0.00\end{array}$ & $\begin{array}{l}\text { 19); }\left.\right|^{2}= \\
\text { 9) }\end{array}$ & $11 \%$ & & & $\begin{array}{ccccc}0.1 & 0.2 & 0.5 & 1 & 2 \\
\text { Favours high Ki-67 } & \text { Favours }\end{array}$ \\
\hline
\end{tabular}

Figure 5. LVI positive rates between high and low Ki-67 expression groups in invasive breast cancer.

cancer with negative ER and PR. ER and PR status were assessed with cut-off value of 10\% positive tumor nuclei according to the previous CAP guideline, 2010. The new CAP guideline for hormone receptor testing recommended that tumors with at least 1\% positive tumor nuclei for ER and/or PR should be designated hormone receptor positive [11], specialist recommended adjuvant endocrine therapy in almost all patients with breast cancer in the presence of any detectable PR/ER [12]. The judgement criterion of the ER, PR status did not accord in the included 5 studies, but merge OR confirmed LVI positive rate was lower in ER positive group than 
that in ER negative group and there was significant difference statistically $(P=0.0010)$.

PR was associated with slow growth, better differentiation, and better overall prognosis. PR positive could enhance the sensitiveness of breast cancer to endocrine therapy significantly. So the St Gallen Meeting (2013) modified the classification criterion [13]. They increased the cut-off value of PR from previous $1 \%$ to current $\geq 20 \%$ in distinguishing "Luminal A-like" from "Luminal B-like" subtypes according to survival differences, and best correspond to Luminal A like subtype [14]. Braun $\mathrm{M}$ et al. adopted different judgement criterion of the PR expression status detected by IHC in the included 4 studies, which led to obvious heterogeneity in PR analysis group $\left(\mathrm{I}^{2}=90 \%\right)$. Therefore the data on PR in [Braun M 2008] was excluded. The result confirmed LVI positive rate was lower in PR positive group than that in PR negative group and there was difference statistically $(P=$ $0.03)$.

HER-2 was an oncogene coding for a tyrosine kinase receptor that activated critical signal transduction pathways resulting in an aggressive phenotype and poor outcome in breast cancer [15], approximately $15 \%$ of patients with breast cancer had the overexpression of the HER-2 protein [16]. Brain metastases were common in patients with HER-2 positive metastatic breast cancer, with up to half of patients experiencing brain metastases. The individual OR value of the 4 studies ranged from 0.76 to 1.98 which indicated that the studies were not consistent about the relation between LVI and HER-2. But the meta-analysis confirmed LVI positive rate was significant lower in HER-2 negative group than that in HER-2 positive group statistically $(P=0.04)$.

Ki-67 was an independent prognostic factor for disease free survival (DFS) in breast cancer [17]. Many studies have investigated the IHC expression of Ki-67 as a predictor for breast cancer [18]. The higher the Ki-67 expressed, the faster the tumor proliferated, the poorer the prognosis was. The choice of the cut-off value had a major impact on practice as it determined which patients were classified as "high Ki-67", and which had a poorer prognosis and should receive more aggressive therapy. The St Gallen Meeting determined that the Ki-67 was chiefly important for distinguishing "luminal A-lke" from "luminal B-like” breast cancer subtypes with a cut-off value of $14 \%$ [19]. The meta-analysis confirmed LVI positive rate was significant higher in high Ki-67 expression group than that in low Ki-67 expression group statistically $(P=0.04)$.

In summary, LVI was significantly correlated with the expression status of ER, PR, HER-2 and Ki-67 in invasive breast cancer and was consistent with poor prognostic status of the four factors and showed an aggressive predictior. Thus, it is considerable to list LVI as a marker of clinical typing for breast cancer, anti-LVI therapy maybe become new therapeutic target for breast cancer.

\section{References}

[1] Siegel, R., Ma, J., Zou, Z. and Jemal, A. (2014) Cancer Statistics, 2014. CA: A Cancer Journal for Clinicians, 64, 9-29. http://dx.doi.org/10.3322/caac.21208

[2] Schoppmann, S.F., Bayer, G., Aumayr, K., Taucher, S., Geleff, S., Rudas, M., et al. (2004) Prognostic Value of Lymphangiogenesis and Lymphovascular Invasion in Invasive Breast Cancer. Annals of Surgery, 240, 306-312. http://dx.doi.org/10.1097/01.sla.0000133355.48672.22

[3] Vleugel, M.M., Bos, R., van der Groep, P., Greijer, A.E., Shvarts, A., Stel, H.V., et al. (2004) Lack of Lymphangiogenesis during Breast Carcinogenesis. Journal of Clinical Pathology, 57, 746-751. http://dx.doi.org/10.1136/jcp.2003.014480

[4] Goldhirsch, A., Glick, J.H., Gelber, R.D., Gelber, R.D., Thürlimann, B., Senn, H.J., et al. (2005) Meeting Highlights: International Expert Consensus on the Primary Therapy of Early Breast Cancer 2005. Annals of Oncology: Official Journal of the European Society for Medical Oncology/ESMO, 16, 1569-1583.

[5] Arnaout-Alkarain, A., Kahn, H.J., Narod, S.A., Sun, P.A. and Marks, A.N. (2007) Significance of Lymph Vessel Invasion Identified by the Endothelial Lymphatic Marker D2-40 in Node Negative Breast Cancer. Modern pathology, 20, $183-191$.

[6] Braun, M., Flucke, U., Debald, M., Walgenbach-Bruenagel, G., Walgenbach, K.-J., Höller, T., et al. (2008) Detection of Lymphovascular Invasion in Early Breast Cancer by D2-40 (Podoplanin): A Clinically Useful Predictor for Axillary Lymph Node Metastases. Breast Cancer Research and Treatment, 112, 503-511. http://dx.doi.org/10.1007/s10549-007-9875-2

[7] Gujam, F.J., Going, J.J., Mohammed, Z.M., Orange, C., Edwards, J. and McMillan, D.C. (2014) Immunohistochemical Detection Improves the Prognostic Value of Lymphatic and Blood Vessel Invasion in Primary Ductal Breast Cancer. BMC Cancer, 14, 676. http://dx.doi.org/10.1186/1471-2407-14-676

[8] Lee, J.A., Bae, J.W., Woo, S.U., Kim, H. and Kim, C.H. (2011) D2-40, Podoplanin, and CD31 as a Prognostic Predictor in Invasive Ductal Carcinomas of the Breast. Journal of Breast Cancer, 14, 104-111.

http://dx.doi.org/10.4048/jbc.2011.14.2.104 
[9] Tezuka, K., Onoda, N., Takashima, T., Takagaki, K., Ishikawa, T., Wakasa, T., et al. (2007) Prognostic Significance of Lymphovascular Invasion Diagnosed by Lymphatic Endothelium Immunostaining in Breast Cancer Patients. Oncology Reports, 17, 997-1003. http://dx.doi.org/10.3892/or.17.5.997

[10] Widodo, I., Ferronika, P., Harijadi, A., Triningsih, F.E. and Soeripto, U.T. (2013) Clinicopathological Significance of Lymphangiogenesis and Tumor Lymphovascular Invasion in Indonesian Breast Cancers. Asian Pacific Journal of Cancer Preventio: APJCP, 14, 997-1001. http://dx.doi.org/10.7314/APJCP.2013.14.2.997

[11] Viale, G., Regan, M.M., Maiorano, E., Mastropasqua, M.G., Dell’Orto, P., Rasmussen, B.B., et al. (2007) Prognostic and Predictive Value of Centrally Reviewed Expression of Estrogen and Progesterone Receptors in a Randomized Trial Comparing Letrozole and Tamoxifen Adjuvant Therapy for Postmenopausal Early Breast Cancer: BIG 1-98. Journal of Clinical Oncology: Official Journal of the American Society of Clinical Oncology, 25, 3846-3852.

[12] Goldhirsch, A., Ingle, J.N., Gelber, R.D., Gelber, R.D., Thürlimann, B., Senn, H.J., et al. (2009) Thresholds for Therapies: Highlights of the St Gallen International Expert Consensus on the Primary Therapy of Early Breast Cancer 2009. Annals of Oncology: Official Journal of the European Society for Medical Oncology/ESMO, 20, 1319-1329.

[13] Goldhirsch, A., Winer, E.P., Coates, A.S., Gelber, R.D., Thürlimann, B., Senn, H.J., et al. (2013) Personalizing the Treatment of Women with Early Breast Cancer: Highlights of the St Gallen International Expert Consensus on the Primary Therapy of Early Breast Cancer 2013. Annals of Oncology: Official Journal of the European Society for Medical Oncology/ESMO, 24, 2206-2223.

[14] Prat, A., Cheang, M.C., Martin, M., Parker, J.S., Carrasco, E., Caballero, R., et al. (2013) Prognostic Significance of Progesterone Receptor-Positive Tumor Cells within Immunohistochemically Defined Luminal A Breast Cancer. Journal of Clinical Oncology: Official Journal of the American Society of Clinical Oncology, 31, 203-209. http://dx.doi.org/10.1200/JCO.2012.43.4134

[15] Slamon, D.J., Clark, G.M., Wong, S.G., Levin, W.J., Ullrich, A. and McGuire, W.L. (1987) Human Breast Cancer: Correlation of Relapse and Survival with Amplification of the HER-2/Neu Oncogene. Science, 235, 177-182. http://dx.doi.org/10.1126/science.3798106

[16] Gonzalez-Angulo, A.M., Litton, J.K., Broglio, K.R., et al. (2009) High Risk of Recurrence for Patients with Breast Cancer Who Have Human Epidermal Growth Factor Receptor 2-Positive, Node-Negative Tumors $1 \mathrm{~cm}$ or Smaller. Journal of Clinical Oncology: Official Journal of the American Society of Clinical Oncology, 27, 5700-5706. http://dx.doi.org/10.1200/JCO.2009.23.2025

[17] Luporsi, E., Andre, F., Spyratos, F., Martin, P.-M., Jacquemier, J., Penault-Llorca, F., et al. (2012) Ki-67: Level of Evidence and Methodological Considerations for Its Role in the Clinical Management of Breast Cancer: Analytical and Critical Review. Breast Cancer Research and Treatment, 132, 895-915. http://dx.doi.org/10.1007/s10549-011-1837-z

[18] Yerushalmi, R., Woods, R., Ravdin, P.M., Hayes, M.M. and Gelmon, K.A. (2010) Ki67 in Breast Cancer: Prognostic and Predictive Potential. The Lancet Oncology, 11, 174-183. http://dx.doi.org/10.1016/S1470-2045(09)70262-1

[19] Goldhirsch, A., Wood, W.C., Coates, A.S., Gelber, R.D., Thürlimann, B., Senn, H.J., et al. (2011) Strategies for Subtypes-Dealing with the Diversity of Breast Cancer: Highlights of the St. Gallen International Expert Consensus on the Primary Therapy of Early Breast Cancer 2011. Annals of Oncology: Official Journal of the European Society for Medical Oncology/ESMO, 22, 1736-1747. 